\title{
Continuous loading of a magnetic trap
}

\author{
J. Stuhlent, P. O. Schmidt*, S. Henslent, J. Wernert, J. Mlynek, and T. Pfaut \\ Fachbereich für Physik, Universität Konstanz, Universitätsstraße 10, D-78457 Konstanz, Germany
}

(October 27, 2018)

\begin{abstract}
We have realized a scheme for continuous loading of a magnetic trap (MT). ${ }^{52} \mathrm{Cr}$ atoms are continuously captured and cooled in a magneto-optical trap (MOT). Optical pumping to a metastable state decouples atoms from the cooling light. Due to their high magnetic moment $\left(6 \mu_{B}\right)$, low-field seeking metastable atoms are trapped in the magnetic quadrupole field provided by the MOT. Limited by inelastic collisions between atoms in the MOT and in the MT, we load $10^{8}$ metastable atoms at a rate of $10^{8}$ atoms/s below $100 \mu \mathrm{K}$ into the MT. Optical repumping after the loading allows us to realize a MT of ground state chromium atoms.
\end{abstract}

32.80.Pj, 34.50.Rk

Since their first realization [1], magnetic traps for neutral atoms have become important and powerful tools for many experiments in atom and quantum optics. Especially striking experimental results [2] have been achieved with Bose-Einstein condensates (BECs) that were realized by evaporatively cooling an atomic gas in a magnetic trap (MT) 3 [- 5 . Up to the present, several groups have demonstrated pulsed [6, [7, quasi-continuous [8] or continuous (cw) [9] outcoupling of magnetically trapped BECs. Although multiply loading of a MT has been achieved 10] experiments so far suffer from the absence of a method for efficient cw loading of atoms into a BEC. Hence to date a matter wave analogon to the continuous wave optical laser has not been realized. Alternatively, a cw atom laser based on magnetic guiding in combination with atomic collisions was suggested [11]. In addition, cw loading of low-dimensional optical traps with laser cooled atoms was proposed [12] and recently demonstrated [13].

In this letter we report on the cw loading of a three dimensional conservative trap with laser cooled atoms that are decoupled from all light fields present. We show that atoms can be optically pumped within a chromium magneto-optical trap (MOT) 14.15] into metastable "dark" states and stored in a MT built up by the quadrupole magnetic field of the MOT. We present results of systematic studies on the loading process and on the lifetime of the MT. Using the cw loading mechanism and a final repumping process we obtain good starting conditions for experiments towards degenerate quantum gases with ground state chromium atoms.

Our cw loading scheme consists of an atomic reservoir and a conservative trap overlapped in space and time. The reservoir is prepared by cooling atoms in a MOT on a transition $|\mathrm{g}\rangle \rightarrow|\mathrm{e}\rangle$ (FIG. 1). A weak decay chan- nel $|\mathrm{e}\rangle \rightarrow|\mathrm{d}\rangle$ allows the transfer of reservoir atoms into an additional long lived and trapped state $|d\rangle$ in which atoms can be accumulated. In our realization low field seeking Zeeman substates of $|\mathrm{d}\rangle$ are trapped in the magnetic quadrupole field of the MOT. The loading can be very efficient if $|\mathrm{d}\rangle$ atoms are decoupled from the MOT light and if their kinetic energy is smaller than the conservative trap depth. A large decay rate branching ratio $\left(\Gamma_{\text {eg }} / \Gamma_{\text {ed }} \gg 1\right)$ assures a steady state MOT in thermal equilibrium and is expected to greatly reduce reabsorption of transfer photons by atoms in the MT [16].

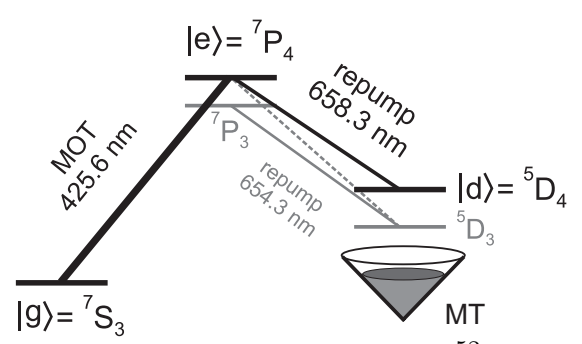

FIG. 1. Relevant part of the ${ }^{52} \mathrm{Cr}$ level scheme. The MOT involves all levels and transitions, the continuous loading process of the magnetic trap (MT) relies on the $\Lambda$-system depicted in black (levels $|\mathrm{g}\rangle,|\mathrm{e}\rangle,|\mathrm{d}\rangle$ ).

Chromium combines the desired $\Lambda$-like level scheme (FIG. 1, black levels and transitions) with a high magnetic moment of up to $6 \mu_{B}$ ( $\mu_{B}=$ Bohr's magneton). Due to its isotopic composition (3 bosons: ${ }^{52} \mathrm{Cr}(84 \%)$, ${ }^{50} \mathrm{Cr}(4 \%),{ }^{54} \mathrm{Cr}(2 \%)$, and one fermion: ${ }^{53} \mathrm{Cr}(10 \%)$ ) it is a promising element for experiments with degenerate atomic Bose and Fermi gases. The magnetic dipoledipole interaction is much stronger than for alkalis and may lead to to a BCS-like transition in a degenerate Fermi gas of ${ }^{53} \mathrm{Cr}[17]$. In addition, chromium has technological potential in nanostructure fabrication [18,19] and structured doping [20] by atom lithography.

Magneto-optical trapping of chromium is performed on the ${ }^{7} \mathrm{~S}_{3} \rightarrow{ }^{7} \mathrm{P}_{4}$ transition (vacuum wavelength $\lambda_{\mathrm{PS}}=$ $425.6 \mathrm{~nm}$, decay rate $\Gamma_{\mathrm{PS}}=31.5 \times 10^{6} \mathrm{~s}^{-1}$, saturation intensity $I_{s}=8.5 \mathrm{~mW} / \mathrm{cm}^{2}$, FIG. 1). Two intercombination lines connect the excited ${ }^{7} \mathrm{P}_{4}$ state to the metastable states ${ }^{5} \mathrm{D}_{4}\left(\lambda_{\mathrm{PD} 4}=658.3 \mathrm{~nm}\right)$ and ${ }^{5} \mathrm{D}_{3}$ $\left(\lambda_{\mathrm{PD} 3}=649.3 \mathrm{~nm}\right)$ [14]. To our knowledge, the lifetime $\tau_{\mathrm{D}}$ of these metastable states has not been measured to date but a lower limit of $\tau_{\mathrm{D}}>50 \mathrm{~s}$ can be deduced from our MT decay times. Measurements of MOT lifetimes give decay rates of $\Gamma_{\mathrm{PD} 4}=(127 \pm 14) \mathrm{s}^{-1}$ and $\Gamma_{\mathrm{PD} 3}=(42 \pm 6) \mathrm{s}^{-1}$ 21]. We effectively reduce the level 
scheme to a $\Lambda$-system $\left(|\mathrm{g}\rangle={ }^{7} \mathrm{~S}_{3},|\mathrm{e}\rangle={ }^{7} \mathrm{P}_{4},|\mathrm{~d}\rangle={ }^{5} \mathrm{D}_{4}\right.$, $\left.\Gamma_{\text {eg }}=\Gamma_{\mathrm{PS}}, \Gamma_{\text {ed }}=\Gamma_{\mathrm{PD} 4}\right)$ with a branching ratio of 250000 by shining in a ${ }^{5} \mathrm{D}_{3} \rightarrow{ }^{7} \mathrm{P}_{3}$ repumper laser. As long as no repumper laser is applied on the $|\mathrm{d}\rangle \rightarrow|\mathrm{e}\rangle$ transition atoms are optically pumped into the $\left|\mathrm{d}, \mathrm{m}_{\mathrm{d}}\right\rangle$ substates $\left(\mathrm{m}_{\mathrm{d}}=-4, \ldots, 4\right.$ denotes the magnetic quantum number) of $|d\rangle$ with a significant probability of ending in low field seeking $\left(\mathrm{m}_{\mathrm{d}}>0\right)$ states.

Our UHV-system consists of two vertically arranged chambers connected by a Zeeman slower. An effusion cell operated at $T_{\mathrm{o}} \sim 1700 \mathrm{~K}$ is attached to the lower chamber. Evacuation by an ion pump and a Ti-sublimation pump leads to residual gas pressures around $10^{-11} \mathrm{mbar}$ in the upper chamber where the traps are located. Three pairs of retroreflected $1 \mathrm{~cm}$ diameter laser beams build up a standard six beam $\sigma^{+} / \sigma^{-}$-light field for the MOT. Two coils wrapped onto the chamber produce a quadrupole magnetic field with gradients up to $b=20 \mathrm{G} / \mathrm{cm}$. The $4 \mathrm{~mm}$ diameter repumper laser beams pass the MOT and are retroreflected. We generate the laser light for the MOT and the Zeeman slower by frequency doubling a Ti:Sapphire laser using a LBO-crystal. Two diode lasers systems serve for repumping on the ${ }^{5} \mathrm{D}_{3} \rightarrow{ }^{7} \mathrm{P}_{3}$ and ${ }^{5} \mathrm{D}_{4} \rightarrow{ }^{7} \mathrm{P}_{4}(|\mathrm{~d}\rangle \rightarrow|\mathrm{e}\rangle)$ transitions (FIG. 11).

We detect trapped metastable atoms by optically pumping them within a few ms back into the ground state $|g\rangle$. Then $|g\rangle$ atoms are resonantly excited with the MOT laser and their fluorescence is imaged onto a calibrated CCD camera. Repumping with the magnetic field on loads the MT with ground state chromium atoms 22. Optical transfer of $|\mathrm{d}\rangle$ atoms into $|\mathrm{g}\rangle$ comes with a heating on the order of the recoil temperature $\left(T_{r} \approx 1 \mu \mathrm{K}\right)$ due to photon scattering. Optical pumping within the magnetic potential may change the mean magnetic moment of trapped atoms and alter their temperature due to a variation in potential energy. However, both effects can be neglected within our experimental resolution since the temperatures exceed $T_{r}$ by more than one order of magnitude and the mean magnetic moment is much larger than its expected change 21]. In the experiments described here the $|\mathrm{d}\rangle \mathrm{MT}$ can therefore be mapped onto the $|\mathrm{g}\rangle$ MT by applying repumping light for a few ms.

We investigated the $\mathrm{cw}$ loading of the MT by performing the following experiments. First we prepare a steady state MOT with both repumper lasers on so that effectively no loading into the MT occurs. Then we switch off the $|\mathrm{d}\rangle$ repumper laser and start $\mathrm{cw}$ loading of the MT. After a variable time delay we detect the $|\mathrm{d}\rangle$ atoms in the MT. The resulting loading curves (number of MT atoms vs. loading time) are well fitted by $N(t)=N_{0}[1-\exp (-t / \tau)]$ (fitting parameters: steady state atom number $N_{0}$, loading time constant $\tau$ ) to extract the MT loading rate $R=N_{0} / \tau$.

Fig. 2 2 shows loading rates for different detunings $(\Delta=$ $\left.\omega_{\text {laser }}-\omega_{\text {atom }}\right)$ of the MOT light and a single laser beam intensity of $15 I_{s}$ vs. the steady state number of atoms in the MOT with open transfer channel. The number of MOT atoms was adjusted by varying $T_{\mathrm{o}}$ and/or the efficiency of the Zeeman slower. For more than a few $10^{7}$ MOT atoms the collisional loss rate reaches the same order of magnitude as $\Gamma_{\text {ed }}$. Even with all repump lasers we trap not more than $10^{8}$ atoms in the MOT [21].

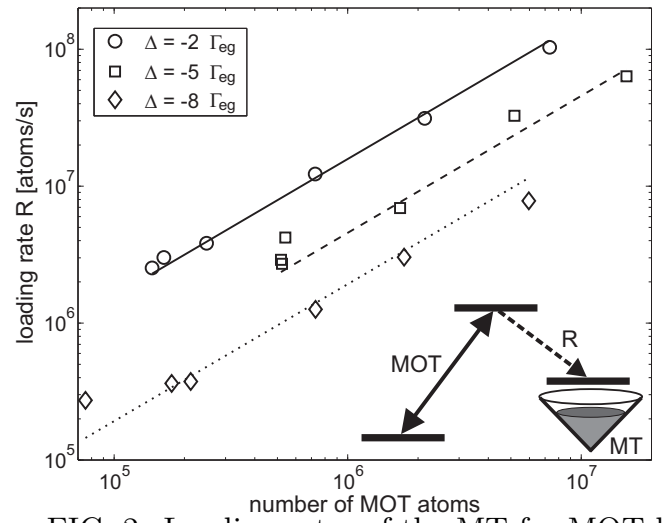

FIG. 2. Loading rates of the MT for MOT laser detunings of $\Delta=-2 \Gamma_{\mathrm{eg}}$ (circles), $-5 \Gamma_{\mathrm{eg}}$ (squares) and $-8 \Gamma_{\mathrm{eg}}$ (diamonds) as a function of the number of atoms in the MOT. The lines are linear least square fits to the data. The marker size represents the accuracy of our measurements.

For given light field parameters $R$ depends linearly on the number of MOT atoms. In order to evaluate the transfer efficiency $\eta=R /\left(N_{\mathrm{MOT}} P_{e} \Gamma_{e d}\right)$ of the loading process, the excitation probability $P_{e}$ was calculated using an averaged saturation intensity of $\left\langle I_{s}\right\rangle=\frac{7}{3} I_{s}$ as in 15.23. We observe $\eta=(32 \pm 5) \%,(25 \pm 4) \%$ and $(16 \pm 4) \%$ for $\Delta=-2,-5,-8 \Gamma_{\text {eg }}$. Loading the MT at rates up to $R=10^{8}$ atoms $/ \mathrm{s}$, we accumulate $10^{8}$ atoms at peak densities of $n_{0}=10^{10}$ atoms $/ \mathrm{cm}^{3}$. Typical $1 / \mathrm{e}-$ radii of the MT are $r \sim 800 \mu \mathrm{m}$ while the radii of the Gaussian shaped MOT are about $\sigma \sim 200 \mu \mathrm{m}$.

The maximum number of atoms in the MT is limited by the loading time constant of $\tau \approx 1 \mathrm{~s}$ observed at high loading rates. FIG. 3 shows the inverse loading time constants for the experimental parameters described above. The decay rates $\Gamma=1 / \tau$ are corrected for "dark" collisions with the residual gas and the thermal chromium beam. This correction is done by subtracting decay rates of the MT in the chromium beam that were measured without MOT laser light and range in the shown data set from $1 / 20 \mathrm{~s}$ to $1 / 2 \mathrm{~s}$ depending on $T_{\mathrm{o}}$. We plot $\Gamma$ vs. the product of the effective density of excited MOT atoms $n_{e}$ times the average collisional velocity $v$. Although measured for different detunings, $\Gamma$ increases linearly with $n_{e} v$ according to

$$
\Gamma=n_{e} \sigma_{\mathrm{ed}} v
$$

with a collisional cross section $\sigma_{\text {ed }}$. This linear dependency shows that if both traps are overlapped inelastic collisions between excited atoms in the MOT and $|\mathrm{d}\rangle$ atoms in the MT are dominating other loss mechanisms. 


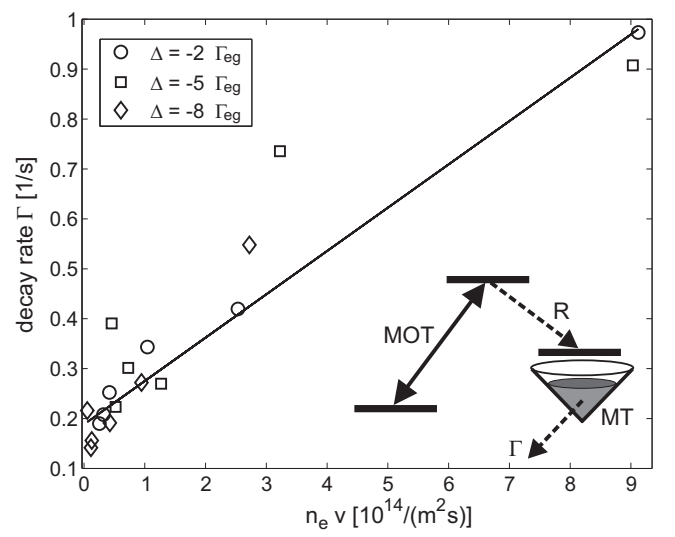

FIG. 3. Decay rates of the MT with overlapped MOT as a function of the effective density of excited MOT atoms $\mathrm{n}_{e}$ times the collisional velocity $v$ for detunings of $\Delta=$ $-2 \Gamma_{\mathrm{eg}}$ (circles), $-5 \Gamma_{\mathrm{eg}}$ (squares) and $-8 \Gamma_{\mathrm{eg}}$ (diamonds). The straight line is a linear fit to the data and gives a cross section for inelastic collisions on zhe order of $\sigma_{\text {ed }} \sim 10^{-15} \mathrm{~m}^{2}$.

Since the MOT is much smaller than the MT collisions occur only at the trap centre and $n_{e}$ can be approximated by the number of excited MOT atoms per volume of the MT 24]. The finite MOT size would give a correction factor on the right hand side of Eq. (11) of 0.5-0.8 depending on the trap size ratio $\sigma / r$. We assume an average collisional velocity of $v \approx\left[\left(T_{\mathrm{MOT}}+T_{\mathrm{MT}}\right) \frac{8 k_{B}}{\pi m_{\mathrm{Cr}}}\right]^{1 / 2}$, where $m_{\mathrm{Cr}}$ is the chromium mass. We extract $\sigma_{\text {ed }} \sim 10^{-15} \mathrm{~m}^{2}$ as an order of magnitude for the cross section of inelastic MOT-MT collisions by fitting the data in FIG. 3 linearly. This value is comparable to the two-body loss rate coefficient in a Cr-MOT 15]. $\sigma_{\text {ed }}$ is about one order of magnitude larger than the values observed in mixtures of two different alkalis [25:26]. Light-assisted collisions with the thermal chromium beam result in a non-vanishing decay rate at very low MOT densities.

The temperature of atoms in the MT is measured in the following way. We pump $|\mathrm{d}\rangle$ atoms back into the ground state $|g\rangle$ with the magnetic field on. Then the $|\mathrm{g}\rangle$ atoms are imaged immediately after switching off the magnetic field. We fit the atomic density distribution to that of a thermal atom ensemble in a quadrupole magnetic field including gravity: $n(x, y, z)=$ $n_{0} \exp \left(-\mathcal{B} \sqrt{x^{2}+y^{2}+4 z^{2}}-\mathcal{G} y\right)$. Here $n_{0}$ is the central density, $\mathcal{B}=\bar{\mu} b /\left(2 k_{B} T\right)$ and $\mathcal{G}=m_{\mathrm{Cr}} g /\left(k_{B} T\right)$. The asymmetry of $n$ along $y$ (vertical axes) due to gravity is clearly visible and the temperature is evaluated from $\mathcal{G}$ with an errorbar of $10 \%$ (fitting accuracy). The mean magnetic moment $\bar{\mu}$ (typically $\bar{\mu}=\mathrm{g}_{\mathrm{d}} \overline{\mathrm{m}}_{\mathrm{d}} \mu_{B}=$ (4.5-6) $\mu_{B}$, corresponding to $\overline{\mathrm{m}}_{\mathrm{d}}=3-4$ ) is determined from the ratio $\mathcal{B} / \mathcal{G}$. The temperature of atoms in the $\mathrm{MT}$ and in the MOT are plotted in FIG. 1 vs. I/ $\Delta$. The MOT temperature, measured by ballistic expansion of the cloud, shows the expected linear increase with $\mathrm{I} / \Delta$ [27]. In the MT we observe temperatures down to $50 \mu \mathrm{K}$ and phase space densities of more than $10^{-7}$.

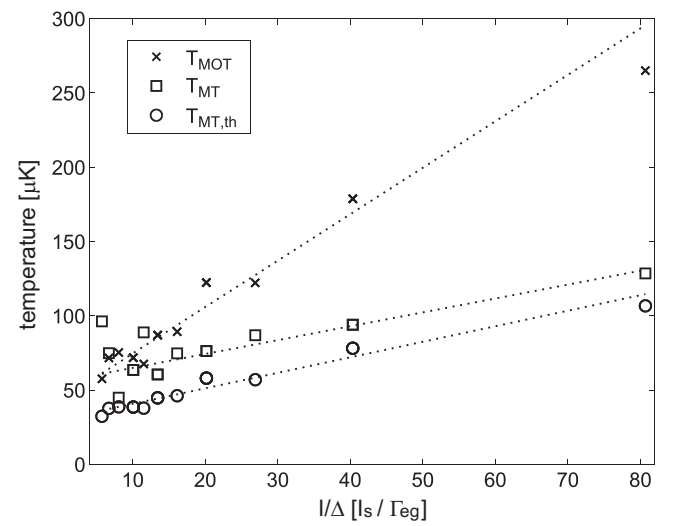

FIG. 4. Temperatures of trapped atoms as a function of the light shift parameter (six-beam intensity $\mathrm{I} / \Delta$ ). $\mathrm{T}_{\mathrm{MOT}}$ (crosses) and $\mathrm{T}_{\mathrm{MT}}$ (boxes) are measured for atoms in the MOT and in the MT, respectively. $\mathrm{T}_{\mathrm{MT}, \mathrm{th}}$ (circles) are the theoretical temperatures of magnetically trapped atoms calculated as described in the text. The lines are linear least square fits to the data.

MT atoms are usually colder than atoms in the MOT. This can be understood by using the Virial Theorem and assuming that the transfer of MOT atoms occurs at the centre of the MT with negligible potential energy. The initial kinetic energy $E_{i}$ of MOT atoms is converted into final kinetic energy $E_{f}$ and potential energy $V_{f}$ (for a linear potential $V_{f}=2 E_{f}$ ) of MT atoms:

$$
\frac{3}{2} k_{B} T_{\mathrm{MOT}}=E_{i}=E_{f}+V_{f}=3 E_{f}=3 \frac{3}{2} k_{B} T_{\mathrm{MT}, \mathrm{th}} .
$$

Including additional initial potential energy due to the finite size of the MOT one gets for the theoretical temperature $T_{\mathrm{MT} \text {,th }}$ of atoms in the MT:

$$
T_{\mathrm{MT}, \mathrm{th}}=\frac{1}{3} T_{\mathrm{MOT}}+\Delta T .
$$

We estimate $\Delta T$ for a transfer from an isotropic MOT with size $\sigma$ into an isotropic MT with mean magnetic field gradient $b$. If $\overline{\mathrm{m}}_{\mathrm{d}}$ is the mean magnetic quantum number of atoms in the MT, $\Delta T$ is given by

$$
\Delta T=\frac{8}{9 \sqrt{2 \pi}} \frac{\mu_{B}}{k_{B}} \mathrm{~g}_{\mathrm{d}} \overline{\mathrm{m}}_{\mathrm{d}} b \sigma
$$

and is about one order of magnitude less than $T_{\mathrm{MOT}}$. Inserting the measured values $T_{\mathrm{MOT}}, \sigma, b$ and $\overline{\mathrm{m}}_{\mathrm{d}}$ in Eqs. (3) and (何) we evaluate the expected $T_{\mathrm{MT} \text {,th }}$ (circles in FIG. (1). Although taking our temperature resolution (about 10\%) into account, atoms in the MT are hotter than theoretically predicted. This effect is more pronounced at low values of $\mathrm{I} / \Delta$ and can be explained by a heating mechanism in the MT. Trapped $|\mathrm{d}\rangle$ atoms are heated by $10-50 \mu \mathrm{K}$ depending on the amount of time spent in the MT and the heating rate described below.

After loading, the number of atoms in the MT decays non-exponentially indicating inelastic two-body collisions 
between $|d\rangle$ atoms. In addition we observe enlargement of the trapped cloud caused by a heating of more than $10 \mu \mathrm{K} / \mathrm{s}$. In contrast, the MT with $|\mathrm{g}\rangle$ atoms decays purely exponentially $\left(N(t) \propto \exp \left(-t / t_{0}\right)\right)$ with a lifetime $t_{0}$ of up to $60 \mathrm{~s}$ and shows heating rates of only $1 \mu \mathrm{K} / \mathrm{s}$. In order to distinguish between the effect of heating and two-body losses in the $|d\rangle$ MT the standard time derivative of the atom density is modified by a term taking the enlargement of the cloud into account:

$$
\frac{d n_{0}}{d t}=-\frac{n_{0}}{t_{0}}-\beta n_{0}^{2}-\frac{n_{0}}{V} \frac{d V}{d t}
$$

where $n_{0}$ is the peak atom density, $V$ the MT volume and $\beta$ the two-body loss rate coefficient. We analyse our data in the following way. The increase of the MT volume $(V=V(t))$ due to heating is fitted linearly. After inserting this $V(t)$ and $t_{0}$ of the ground state MT we solve Eq. (5) for $n_{0}(t)$ and fit the resulting function (fitting parameter $\beta$ ) to the peak density of atoms in the MT. This results in $\beta \sim 7 \times 10^{-17} \mathrm{~m}^{3} / \mathrm{s}$ relatively insensitiv to $t_{0}$. The corresponding cross section $\sigma_{\mathrm{dd}}$ is about one order of magnitude less than $\sigma_{\text {ed }}$. Since the product of the mean MT atom density times $\beta$ is about one order of magnitude less than the inverse loading time constant inelastic $|d\rangle-|d\rangle$ collisions do not limit the number of atoms in the cw loaded MT. To date we are not able to resolve which one of the possible mechanisms - magnetic field instabilities, Majorana transitions, spin flip collisions that release Zeeman energy or small angle collisions with atoms that leave the MT after a state changing collision - is dominant for the observed heating rates. The first two processes are expected to be suppressed in a new MT with non-zero field minimum. In addition, full atomic polarization in the extreme Zeeman substate should lead to a reduction of $\beta$ as predicted for $\mathrm{He}^{*}$ [28].

In summary, we have studied a new continuous optical loading scheme for conservative atom traps based on the operation of an atomic reservoir (here a MOT) and a conservative trap (here a MT) overlapped in space and time. MOT atoms are transferred to the MT by a spontaneous decay into metastable states that are decoupled from both MOT light and transfer photons. The loading rates up to $10^{8}$ atoms/s depend linearly on the number of excited MOT atoms. We continuously load up to $10^{8}$ atoms into the MT, limited by collisions with excited MOT atoms. The lifetime of metastable atoms in the MT after switching off the MOT is currently limited by inelastic trapped atom collisions that are strongly suppressed using ground state atoms. In future experiments, the cw loading of different kinds of magnetic traps (TOP [29] - and optical plug [5] trap) and the observed heating will be investigated. Initial phase space densities on the order of $10^{-7}$ of $10^{8}$ ground state chromium atoms obtained by cw loading and a final repumping process encourage work towards Bose-Einstein condensation.
This work was financed by the Deutsche Forschungsgemeinschaft and the Optik Zentrum Konstanz. P. O. Schmidt is supported by the Studienstiftung des deutschen Volkes.

* Present Address: 5. Physikalisches Institut, Universität Stuttgart, Pfaffenwaldring 57, D-70550 Stuttgart, Germany.

[1] A. L. Migdall et al., Phys. Rev. Lett. 54, 2596 (1985).

[2] Bose-Einstein Condensation in Atomic Gases, Vol. 140 of Proceedings of the International School of Physics Enrico Fermi, edited by M. Inguscio, S. Stringari, and C. E. Wieman (IOS Press, Amsterdam, 1999).

[3] M. H. Anderson et al., Science 269, 198 (1995).

[4] C. C. Bradley, C. A. Sackett, J. J. Tollett, and R. G. Hulet, Phys. Rev. Lett. 75, 1687 (1995).

[5] K. B. Davis et al., Phys. Rev. Lett. 75, 3969 (1995).

[6] M.-O. Mewes et al., Phys. Rev. Lett. 78, 582 (1997).

[7] J. L. Martin et al., J. Phys. B: At. Mol. Opt. Phys. 32, 3065 (1999).

[8] E. W. Hagley et al., Science 283, 1706 (1999).

[9] I. Bloch, T. W. Hänsch, and T. Esslinger, Phys. Rev. Lett. 82, 3008 (1999).

[10] E. A. Cornell, C. Monroe and C. E. Wieman, Phys. Rev. Lett. 67, 2439 (1991).

[11] E. Mandonnet et al., Eur. Phys. J. D 10, 9 (2000).

[12] T. Pfau and M. Mlynek, TOPS Bose-Einstein Condensation 7, 33 (1997).

[13] D. Schneble et al., IQEC post deadline paper, technical digest CLEO/EUROPE-IQEC 2000.

[14] A. S. Bell et al., Europhys. Lett. 45, 156 (1999).

[15] C. C. Bradley, J. J. McClelland, W. R. Anderson, and R. J. Celotta, Phys. Rev. A 61, 053407 (2000).

[16] L. Santos, F. Floegel, T. Pfau, and M. Lewenstein, quant$\mathrm{ph} / 0007003$.

[17] M. Houbiers and H. T. C. Stoof, Phys. Rev. A 59, 1556 (1999).

[18] U. Drodofsky et al., Appl. Phys. B 65, 755 (1997).

[19] J. J. McClelland, R. E. Scholten, E. C. Palm, and R. J. Celotta, Science 262, 877 (1993).

[20] T. Schulze et al., Appl. Phys. Lett., in press.

[21] J. Stuhler, Ph.D. thesis, Universität Konstanz (2001).

[22] This is an alternative method to the buffer gas loading of J. Weinstein et al., Phys. Rev. A 57, 3173 (1998).

[23] M. J. Snadden, A. S. Bell, R. B. M. Clarke, and E. Riis, J. Opt. Soc. Am. B 14, 544 (1997).

[24] We define the volume $V$ of an isotropic MT with 1/eradius $r$ by $V=8 \pi r^{3}$.

[25] G. D. Telles et al., Phys. Rev. A 59, (1999).

[26] U. Schlöder et al., Eur. Phys. J. D 7, 331 (1999).

[27] C. D. Wallace et al., J. Opt. Soc. Am. B 11, 703 (1994).

[28] G. V. Shlyapnikov, J. T. M. Walraven, U. M. Rahmanov, and M. W. Reynolds, Phys. Rev. Lett. 73, 3247 (1994).

[29] W. Petrich, M. H. Anderson, J. R. Ensher, and E. A. Cornell, Phys. Rev. Lett. 74, 3352 (1995). 